\title{
MODELISATION NUMÉRIQUE DU COURANT ET DU TRANSPORT SÉDIMENTAIRE INDUITS PAR LE DÉFERLEMENT
}

\author{
P. PECHON, C. TEISSON
}

Laboratoire National d'Hydraulique - EDF

\begin{abstract}
The numerical modelling of wave height decay, timeaveraged $3 D$ currents and sediment transport in the surf-zone is briefly described. Numerical results are compared with measurements on two cases conducted in flumes. These tests show that the hydrodynamic model gives satisfying results and the compound sedimentological system can reproduce the generation and the off-shore migration of a bar due to breaking waves.

Moreover the computation of wave-driven currents near a detached breakwater reveals the threedimensional structure of the circulation. The depthintegrated flow pattern displays two symetrical large eddies whereas the velocities at the bottom tends to converge towards the centres of the eddies.
\end{abstract}

\section{INTRODUCTION}

Les courants engendrés par la houle comptent parmi les principaux facteurs hydrodynamiques d'évolution du littoral, et les mécanismes de leur action sur une plage sont complexes. On distingue ainsi :

- Les courants tridimensionnels, moyennés sur la période de houle, induits par le déferlement. En période de tempête, ils sont très érosifs et provoquent d'importants transits de sable parallèlement à la côte. Ils remanient également le profil de plage en faisant descendre le sédiment du haut de plage vers la ligne de déferlement.

- Les vitesses orbitales de la houle. Sinusoïdales au large, elles deviennent dissymétriques à l'approche du rivage, soit par le raidissement du front d'onde, soit par les effets non-linéaires (courant d'expansion lié à la diffraction, ...). Lorsque la houle a une grande période et une faible hauteur, la dissymétrie des vitesses orbitales contribue à remonter le matériau du large vers la côte.

- Le mouvement de va-et-vient des vagues le long du rivage. Ce phénomène favorise les échanges entre la zone émergée et la zone immergée. Le rechargement du haut de plage, le sapement des dunes ou le mouvement de translation d'un cordon littoral par le franchissement proviennent du transport au rivage. Il faut remarquer que les fluctuations saisonnières de la ligne de rivage peuvent être bien supérieures aux évolutions à l'échelle de lannée. De ce point de vue, ce transport joue aussi un rôle important puisqu'il remanie le profil de plage.

- Les courants moyens dans la couche-limite. Ils sont mal connus mais des observations en canal expérimental ont montrés qu'ils avaient un rôle significatif en dehors de la zone de déferlement.

Le présent article concerne le premier type de transport évoqué ci-dessus, relatif aux transits dans la zone de déferlement.
Les modèles numériques ont d'abord permis de calculer le transport littoral dû aux courants engendrés par le déferlement, exprimés sous forme moyennée sur la profondeur d'eau (Horikawa, 1988, Southgate, 1989, Péchon, 1987). Ces codes bidimensionnels fournissent des informations interessantes puisque c'est le transport littoral qui prédomine en général, en particulier lorsqu'on examine les évolutions sédimentaires à une échelle globale. Par contre, ils ne permettent pas d'obtenir les évolutions détaillées au voisinage immédiat d'un ouvrage par exemple, puisqu'ils ne prennent pas en compte l'hétérogénéité du profil vertical de vitesse.

Des modèles bidimensionnels verticaux, applicables au remaniement du profil de plage, ont été proposés par Svendsen et Hansen (1988), Fredsoe et Deigaard (1991).

Les modèles horizontaux et verticaux ont été combinés de façon non couplée par de Vriend, 1987 et Sanchez Arcilla (1990) pour aboutir aux modèles dits quasi-3D. Plus complète que les approches précédentes, la modélisation quasi-3D ne permet cependant pas d'apprécier la direction des courants près du fond pour des bathymétries complexes à cause du découplage entre la structure horizontale et la structure verticale de l'écoulement.

Dans la modélisation décrite ici, l'écoulement est calculé à l'aide du code TELEMAC-3D en résolvant les équations tridimensionnelles de l'hydraulique moyennées en temps et en couplant les trois composantes de vitesse. Le transport sédimentaire est déduit du champ de courant près du fond puis les évolutions de la bathymétrie sont déterminées.

\section{LA MODÉLISATION NUMÉRIQUE}

\subsection{Généralités}

La simulation des mouvements du fond résulte d'un enchaînement de calculs :

- propagation de la houle du large vers la côte et déferlement de la houle,

- courants tridimensionnels engendrés par le déferlement,

- transport sédimentaire et évolution du fond.

Lorsque les variations du fond sont importants, l'interaction avec l'écoulement est prise en compte en recalculant la houle et le courant pour la nouvelle bathymétrie.

\subsection{Le modèle de houle}

Ce code (Péchon, 1987) simule la réfraction en supposant que la houle suit la théorie non-linéaire de Serre (1953). Il y a déferlement lorsque le rapport $\mathrm{H} / \mathrm{h}$, où $\mathrm{H}$ est la hauteur de houle et $\mathrm{h}$ la hauteur d'eau, est supéricur à un coefficient $\mathbf{j}$ dépendant de la pente de 
fond et de la cambrure de la houle (Battjes, 1974), compris entre 0,8 et 1,2 .

La hauteur de houle dans le déferlement est obtenue en exprimant que la dissipation d'énergie est similaire à celle d'un ressaut hydraulique (Lamb, 1932, Stive, 1984).

\subsection{Le modèle de courant dans le déferlement}

On se borne ici au calcul du courant moyen en temps et induit par le deferlement. Les vitesses instantanées de la houle et le courant de masse qui ne sont pas dus au déferlement seront obtenus à partir des résultats du modèle de houle.

Le champ de courant est issu de la résolution des équations moyennées en temps à l'aide du code TELAMAC-3D fondé sur une méthode numérique aux éléments finis (Lepeintre et al., 1991). L'idée de base consiste à spécifier une distribution de force suivant la colonne d'eau qui comprend deux parties :

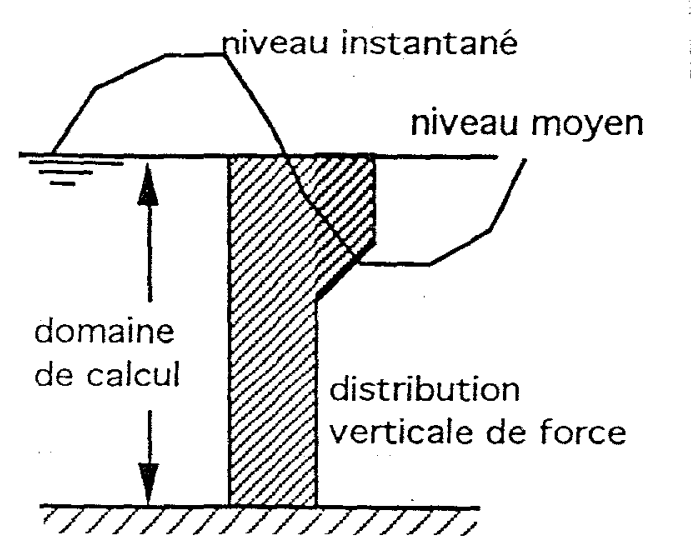

- Une composante constante sur la verticale représente les variations de flux de quantité de mouvement dans le déferlement pour la vitesse "organisée" de la houle (Longuet Higgins, 1970).

- Une composante représentant l'entrainement de la masse d'eau par les brisants. Ce terme est non nul dans la partie supérieure de la colonne d'eau, sur une épaisseur égale à la demi-hauteur de houle.

\subsection{Le modèle de transport sédimentaire}

Dans le cas d'une plage de sable, le volume de matériau transporté par un courant superposé à la houle peut être estimé à partir de la formule de Bailard (1981). Celle-ci fournit en réalité deux valeurs, l'une pour le transport par charriage qui s'exerce près du fond, l'autre pour le débit solide en suspension. La formule de Bailard a l'avantage de faire intervenir les moments de la vitesse totale instantanée près du fond, de la forme $|\vec{u}|^{n} \vec{u}$, ce qui permet notamment de rendre compte de l'asymetrie de la houle.

Par contre, elle a été établie pour une houle non déferlante. Pour représenter l'effet de remise en suspension plus intense dans le déferlement, le transport en suspension donné par la formule initiale est multiplié par un facteur $(1+a U / \sqrt{g h})$, U étant la vitesse près du fond due au deferlement, $h$ la hauteur d'eau et a est un coefficient. Dans l'application du paragraphe 3.3, a prend la valeur 20 après ajustement.

Les évolutions du fond sont ensuite déterminées à partir de l'équation de conservation du matériau .

\section{APPLICATIONS}

\subsection{Circulation dans un canal}

Déjà étudié sur modèle physique par Buhr-Hansen and Svendsen (1984), le canal a un fond horizontal au large, de cote $-0,34 \mathrm{~m}$ par rapport au niveau au repos, puis a une pente constante égale à $1 / 36$ jusqu'à la cote $-0,11 \mathrm{~m}$ et se termine par un profil parabolique dont le sommet atteint $-0,07 \mathrm{~m}$ (fig. 1).

En vue de valider le modèle de courant, le champ de hauteur de houle dans le déferlement, imposée en donnée dans le code courantologique, est celui mesuré directement sur le modèle. La hauteur de houle au point de déferlement est de $0,17 \mathrm{~m}$ et la hauteur d'eau au repos est égale à $0,20 \mathrm{~m}$. La figure 1 présente la comparaison du profil de vitesse mesuré suivant deux verticales et du profil calculé. La vitesse totale calculée est obtenue par la somme de la vitesse moyenne due au déferlement et de la vitesse orbitale issue de la théorie cnoïdale, moyennée sur la période de la houle. On observe que la vitesse totale calculée est proche de celle mesurée dans la moitié inférieure de la colonne d'eau, qui nous intéresse pour les problèmes sédimentologiques. La partie supérieure est moins bien reproduite, ce qui est normal car la couche supérieure dans le modèle est relativement artificielle.

\subsection{Formation d'une barre de déferlement}

Des essais d'évolution de profil de plage sous l'action de la houle ont été menés dans un canal à grande échelle à l'Université de Hanovre (Dette et Uliczka, 1986), de 7 mètres de profondeur.

A l'état initial, le profil mis èn place dans l'installation comportait au large une pente de 1/20 prolongée par une pente de $1 / 4$ depuis la cote $-1,0 \mathrm{~m}$ à la cote $+2,0 \mathrm{~m}$, et se terminait par une plate-forme horizontale. Pour commencer la simulation numérique avec une bathymétrie réaliste, le profil initial du domaine modélisé a été calqué sur la bathymétrie relevée dans le canal après 0,8 heures d'action de la houle. Une houle régulière a êté imposée, de hauteur au large égale à 1,5 $\mathrm{m}$ et de période $6,0 \mathrm{~s}$.

Les bathymétries calculées et relevées sur modèle physique sont reportées sur la figure 2 . Le modèle numérique reproduit correctement la création et la migration vers le large de la barre de déferlement mais il ne redonne pas l'approfondissement derrière la barre. 

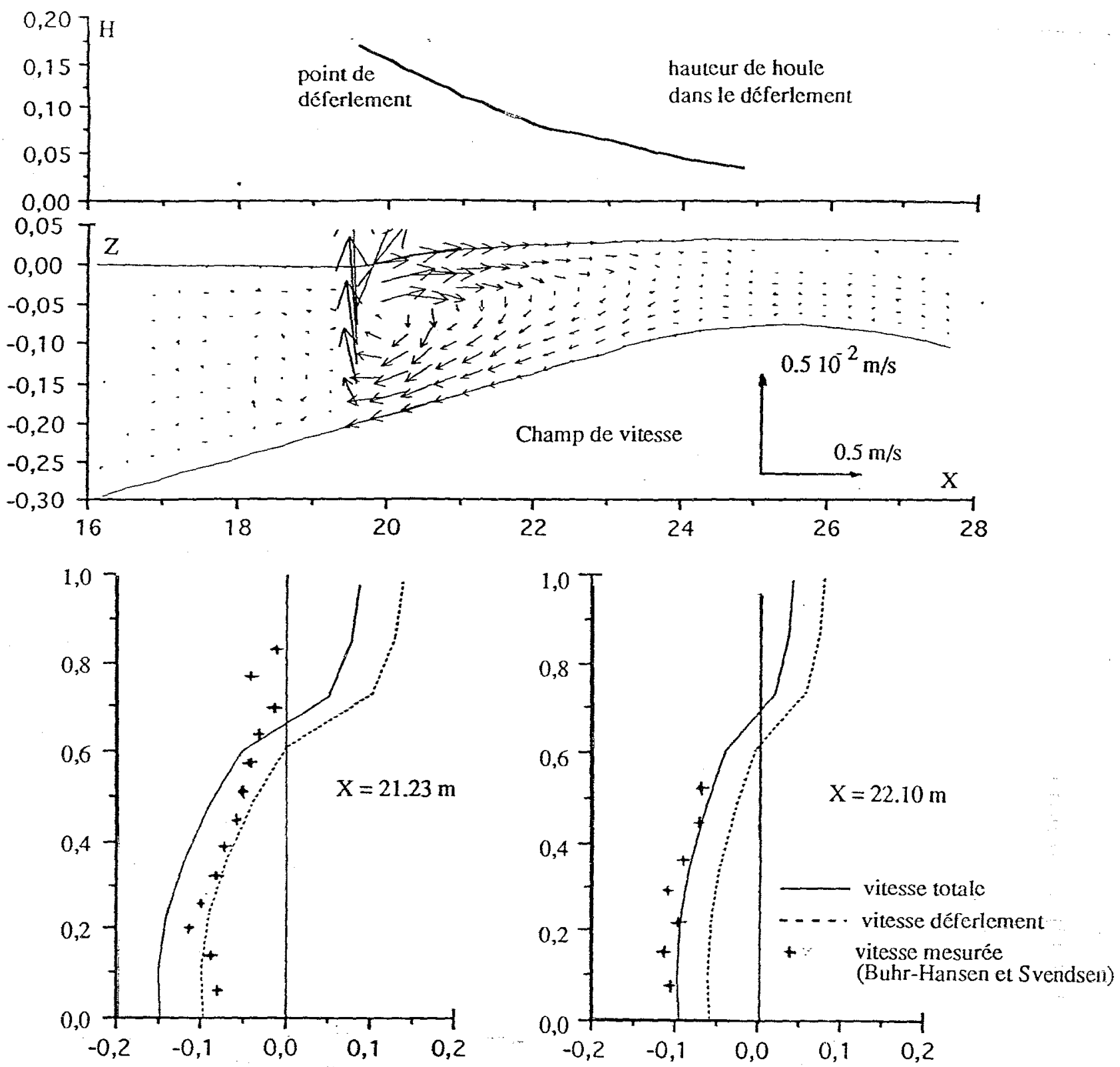

Fig. 1 Courants moyens dans un canal à houle Comparaison des vitesses mesurées et calculées

Celui-ci est dû à l'impact de la houle plongeante qui n'est pas traduit dans la formule de transport.

\subsection{Ecoulement tridimensionnel derrière un brise-} lames

Ce cas a été défini dans le but de reproduire la formation d'un tombolo à l'abri d'un brise-lames. Cette application est actuellement en cours et seul les courants ont été calculés pour le moment, le champ de houle déferlante ayant été grossièrement estimé. L'intérêt de ces résultats encore qualitatifs est de mettre en évidence l'hétérogénéité verticale de l'écoulement. Dans le cadre du projet européen MAST G8M, d'autres instituts traiteront ce type d'ouvrage et les résultats de chacun seront confrontés.

Le domaine étudié fait $600 \mathrm{~m}$ de long et $150 \mathrm{~m}$ de large (fig.3). Le fond a une pente constante de $3 / 100$; la hauteur d'eau au repos est de $5,0 \mathrm{~m}$ au large et de $0,5 \mathrm{~m}$ à la côte. Le brise-lames a une longueur de $100 \mathrm{~m}$ et il est situé à $100 \mathrm{~m}$ du rivage, à une profondeur de $3,5 \mathrm{~m}$. Comme dans le cas précédent, seule la frontière au large est ouverte. La houle entrant dans le domaine est perpendiculaire à la frontière maritime, sa hauteur est égale à 2,50 $\mathrm{m}$ et sa période à $8,0 \mathrm{~s}$.

Pour ce calcul préliminaire, la valeur de la hauteur de houle dans le déferlement est donnée par une fonction analytique, dépendant de la distance à la ligne de déferlement située ici à $100 \mathrm{~m}$ du rivage est nulle à l'abri de l'ouvrage.

La figure 3 montre le champ de vitesses moyennées sur la verticale obtenu à partir des résultats tridimensionnels. Deux larges tourbillons s'établissent derrière le brise-lames. Ils sont principalement générés par les gradients de surface libre car les contraintes dues à la houle déferlante créent une surélévation du niveau en zone ouverte tandis qu'elles sont nulles derrière l'ouvrage.

Des trajectoires de particules dans le champ moyenné sur la verticale et en surface confirment ces larges 

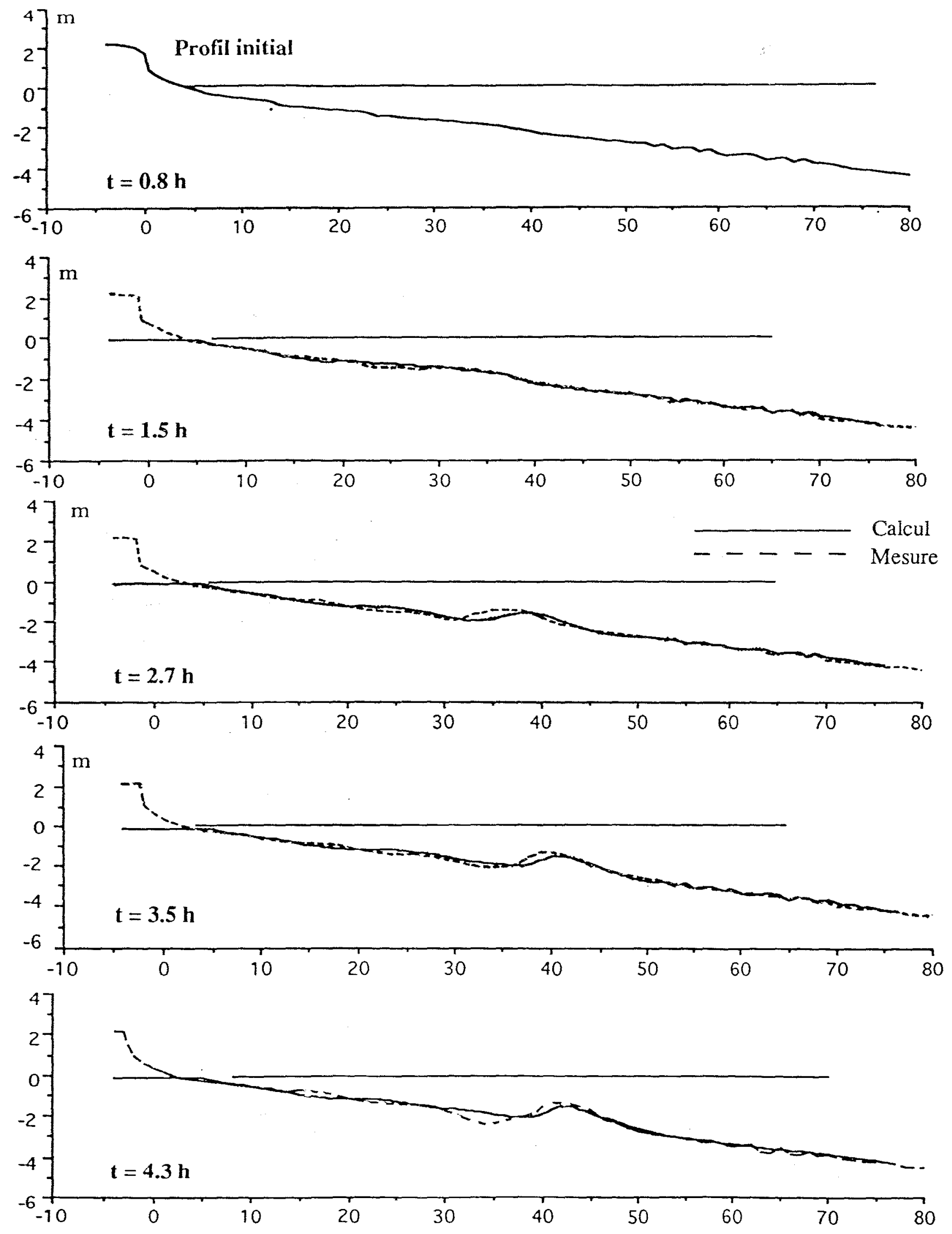

Fig. 2 -Evolution d'un profil de plage

Comparaison des fonds mesurés et calculés 


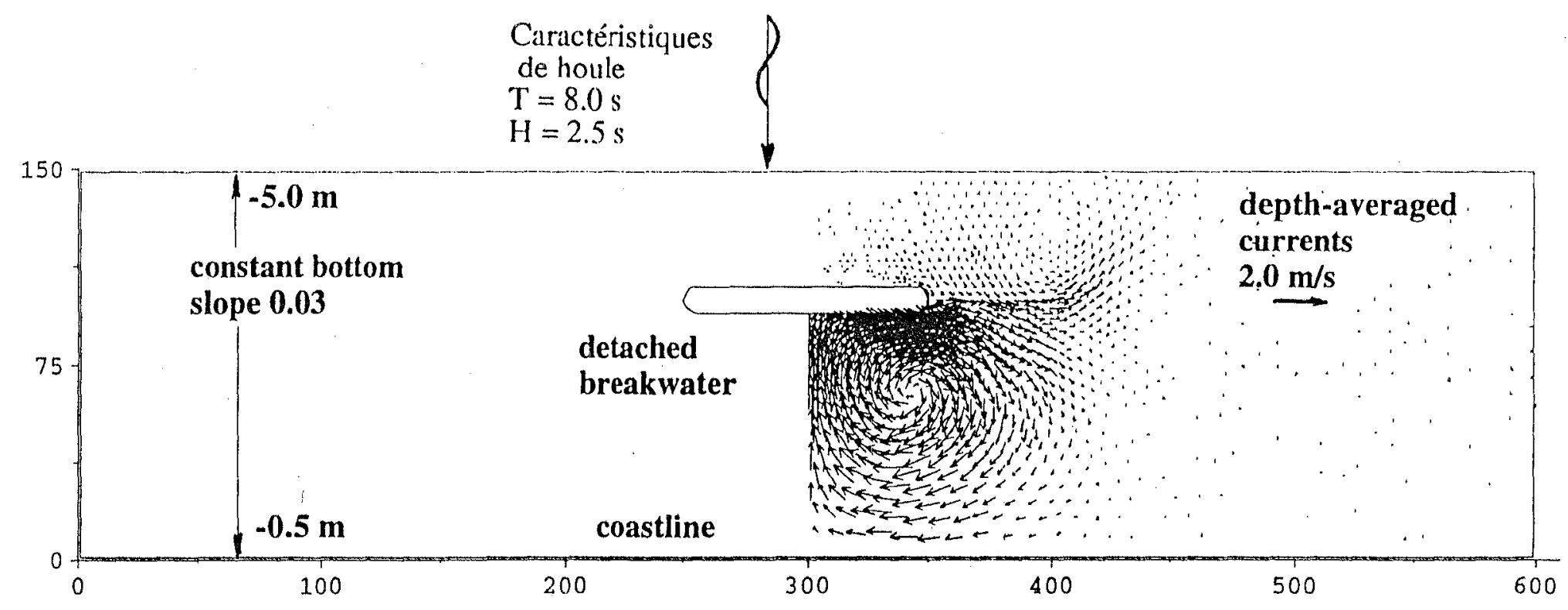

Trajectoires de particules $-200 \mathrm{~s}$
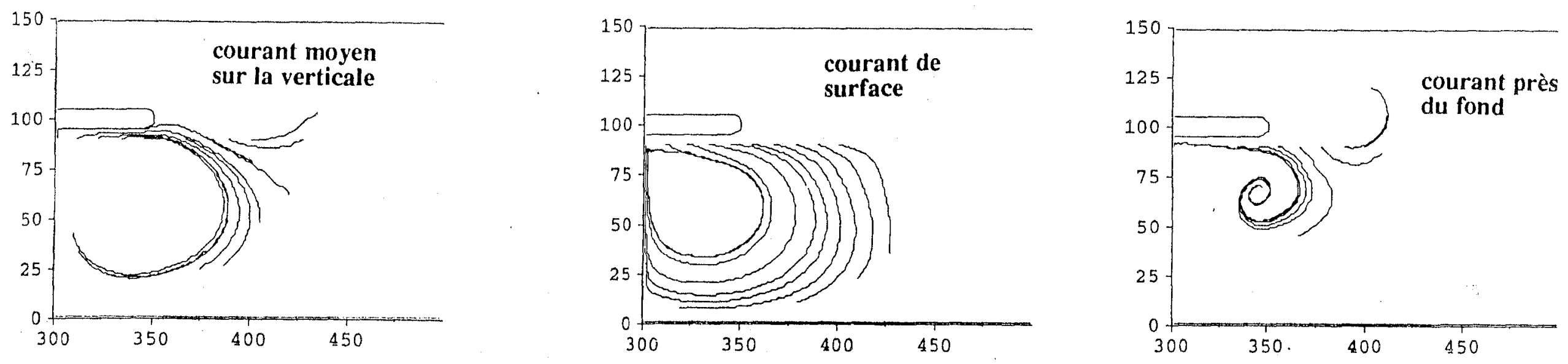

Fig. 3 Courants derriere un brise-lames 
structures tourbillonnaires tandis que les trajectoires près du fond s'orientent préférentiellement vers le centre du tourbillon. Par conséquent, les vitesses agissant sur les particules de sable qui tapissent le fond ne sont absolument pas colinéaires aux vitesses moyennées sur la verticale.

\section{DÉVELOPPEMENTS FUTURS}

Les applications à des cas schématiques donnent des résultats satisfaisants. Cependant, des tests de validation complémentaires doivent encore être menés, notamment dans des cas tridimensionnels.

Le champ de houle peut être complexe dans les applications concrètes et sa reproduction nécessite de mettre en oeuvre un modèle de houle sophistiqué, en particulier lorsque les résultats sont destinés à la détermination du transport sédimentaire. Dans ce but, le LNH développe actuellement un modèle basé sur les équations de Serre dans la bibliothèque TELEMAC, qui permettra de représenter l'ensemble des phénomènes de propagation et de déformation de la houle, en particulier la dissymétric de la surface libre et le mouvement de va et-vient au rivage.

Enfin des travaux menés en collaboration avec d'autres instituts européens sont en cours pour améliorer l'estimation du transport solide en fonction des grandeurs hydrodynamiques. La compréhension du processus d'entraînement des grains de sable s'appuie à la fois sur des essais sur modèle physique et sur des modélisations fines de la couche de fluide près du fond.

En conclusion, il reste encore du travail à faire, mais le caractère opérationnel des modèles numériques appliqués à la sédimentologie devient plus évident, et on est assuré qu'ils seront d'un usage courant dans les années à venir si le potentiel de recherche et développement est maintenu.

\section{Remerciements}

Ces travaux ont été réalisés dans le cadre du programme européen de recherche MAST G8M. Ils ont été financés en partie par le Service Central Technique du Secrétariat d'Etat à la Mer et la Commission des Communautés Européennes, Direction Générale pour la Science, la Recherche et le Développement, sous le contrat $n^{\circ}$ MAS2-CT92-0027.

\section{Références}

Bailard, J.A., An energetic total load sediment transport model for plane sloping beach. J. Geophys. Res., Vol. 86, No. CII, 1991.

Buhr-Hansen, J. and Svendsen, I.A., A theoritical and experimental study of undertow. International Conference on Coastal Engineering, Houston, 1984.

Dette, H., Uliczka, K. Seegangsserzeugte wechselwirkung zwishen vorlang und vorstand sowie küstenschutzbauwerk. Technisher bericht Nr. 3- SBF 205/TP A6. Universität Hannover, 1986.

De Vriend H.J. and Stive M.J.F. Quasi-3D modelling of nearshore currents. Coastal Engineering, 11, $5 \& 6$, p. $565-601,1987$.
Fradsoe, J. and Deigaard, R., Mechanics of Coastal Sediment Transport. World Scientific, 1992.

Horikawa, K., Nearshore Dynamics and Coastal processes. University of Tokio Press, 1988.

Lamb, H., Hydrodynamics. Cambridge University Press. 6th ed., 1932.

Lepeintre, F., Janin, J.M., Péchon, P. TELEMAC-3D : A finite element code to solve $3 D$ free surface flow problems. International Conference of Seas and Coastal Regions, Southampton, 1991.

Péchon, P. Modelling of longshore currents with a non-linear wave theory. ASCE Speciality Conference on Coastal Hydrodynamics, Delaware, 1987.

Serre, F. Contribution à l'étude des écoulements permanents et variables dans les caraux. La Houille Blanche, p. 374-388, p. 830-872, 1953.

Southgate, H., A nearshore profile model of wave and tidal current interaction. Coastal Engineering, Vol. 13, p. 219-245, 1989.

Stive, M.J.F. and Wind, H.G. A study of radiation stress and set-up in the nearshore region. Coastal Engineering, 6, p. 1-25, 1982.

Svendsen, I.A. and Hansen, J.B. cross-shore currents in surf zone modelling. Coastal Engineering, 12, p. $23-$ $42,1988$.

Svendsen, I.A. and Lorenz, R.S. Velocities in combined undertow and longshore currents. Coastal Engineering, 13, p. 55-79, 1989. 